\title{
Biomarker-predicted sugars intake compared with self-reported measures in US Hispanics/Latinos: results from the $\mathrm{HCHS} / \mathrm{SOL}$ SOLNAS study
}

\author{
JM Beasley ${ }^{1, *}, M$ Jung $^{2}, N_{\text {Tasevska }}{ }^{3}$, WW Wong ${ }^{4}$, AM Siega-Riz ${ }^{5}$, D Sotres-Alvarez ${ }^{6}$, \\ MD Gellman ${ }^{7}$, JR Kizer ${ }^{8}$, PA Shaw ${ }^{9}$, J Stamler ${ }^{10}$, M Stoutenberg ${ }^{11}$, L Van Horn ${ }^{10}$, \\ AA Franke ${ }^{12}$, J Wylie-Rosett ${ }^{8}$ and Y Mossavar-Rahmani ${ }^{8}$ \\ 'Department of Medicine, NYU School of Medicine, 550 First Avenue, OBV-CD 673, New York, NY 10016, USA: \\ ${ }^{2}$ Department of Epidemiology, Johns Hopkins School of Public Health, Baltimore, MD, USA: ${ }^{3}$ School of Nutrition and \\ Health Promotion, Arizona State University, Phoenix, AZ, USA: ${ }^{4}$ US Department of Agriculture/Agricultural Research \\ Service Children's Nutrition Research Center, Department of Pediatrics, Baylor College of Medicine, Houston, TX, \\ USA: ${ }^{5}$ Departments of Epidemiology and Nutrition, Gillings School of Global Public Health, University of North \\ Carolina at Chapel Hill, Chapel Hill, NC, USA: ${ }^{6}$ Collaborative Studies Coordinating Center, Department of \\ Biostatistics, Gillings School of Global Public Health, University of North Carolina at Chapel Hill, Chapel Hill, NC, \\ USA: ${ }^{7}$ Department of Psychology, Behavioral Medicine Research Center, University of Miami Miller School of \\ Medicine, Miami, FL, USA: ${ }^{8}$ Department of Epidemiology and Population Health, Albert Einstein College of \\ Medicine, Bronx, NY, USA: ${ }^{9}$ Department of Biostatistics and Epidemiology, University of Pennsylvania Perelman \\ School of Medicine, Philadelphia, PA, USA: ${ }^{10}$ Department of Preventive Medicine, Northwestern University Feinberg \\ School of Medicine, Chicago, IL, USA: " 'Department of Public Health Sciences, University of Miami Miller School of \\ Medicine, Miami, FL, USA: ${ }^{12}$ University of Hawaii Cancer Center, Honolulu, HI, USA
}

Submitted 28 July 2015: Final revision received 25 April 2016: Accepted 12 May 2016: First published online 24 June 2016

\begin{abstract}
Objective: Measurement error in self-reported total sugars intake may obscure associations between sugars consumption and health outcomes, and the sum of $24 \mathrm{~h}$ urinary sucrose and fructose may serve as a predictive biomarker of total sugars intake. Design: The Study of Latinos: Nutrition \& Physical Activity Assessment Study (SOLNAS) was an ancillary study to the Hispanic Community Health Study/Study of Latinos (HCHS/SOL) cohort. Doubly labelled water and $24 \mathrm{~h}$ urinary sucrose and fructose were used as biomarkers of energy and sugars intake, respectively. Participants' diets were assessed by up to three $24 \mathrm{~h}$ recalls ( $88 \%$ had two or more recalls). Procedures were repeated approximately 6 months after the initial visit among a subset of ninety-six participants.

Setting: Four centres (Bronx, NY; Chicago, IL; Miami, FL; San Diego, CA) across the USA.

Subjects: Men and women ( $n$ 477) aged $18-74$ years.

Results: The geometric mean of total sugars was 167.5 (95\% CI 154.4, 181.7) g/d for the biomarker-predicted and $90.6(95 \%$ CI 87.6, 93.6) $\mathrm{g} / \mathrm{d}$ for the self-reported total sugars intake. Self-reported total sugars intake was not correlated with biomarker-predicted sugars intake $(r=-0 \cdot 06, P=0 \cdot 20, n$ 450). Among the reliability sample ( $n$ 90), the reproducibility coefficient was 0.59 for biomarker-predicted and $0 \cdot 20$ for self-reported total sugars intake.

Conclusions: Possible explanations for the lack of association between biomarkerpredicted and self-reported sugars intake include measurement error in self-reported diet, high intra-individual variability in sugars intake, and/or urinary sucrose and Uinury sucres fructose may not be a suitable proxy for total sugars intake in this study population. Urinary sucrose and fructose biomarkers
\end{abstract}

According to the American Heart Association, excessive dietary sugars intake, especially in the form of fructose consumption, may contribute to obesity, insulin resistance, type 2 diabetes, hypertension and dyslipidaemia ${ }^{(1)}$. Possible pathways potentially explaining the role of dietary sugars in increasing cardiometabolic risk include: (i) excess energy 
intake; and/or (ii) high dietary glycaemic load leading to inflammation, insulin resistance and impaired $\beta$-cell function $^{(1,2)}$. US Hispanics/Latinos are 25 (95\% CI 13, 38) \% more likely to report sugar-sweetened beverage consumption than non-Hispanic/Latino adults according to data from the National Health and Nutrition Examination Survey $2007-2008^{(3)}$. Type 2 diabetes is highly prevalent among Hispanics/Latinos in the USA, with wide variability based on Hispanic/Latino background, ranging from $10 \cdot 2 \%$ in South Americans to $18.3 \%$ in Mexicans $(P<0.0001)^{(4)}$.

Measurement error in self-reported intake has impeded progress in definitively addressing diet-disease hypotheses ${ }^{(5-9)}$. Identified strategies for mitigating measurement error include statistical approaches that combine two dietary assessment approaches (e.g. FFQ and $24 \mathrm{~h}$ recall (24HR) or biomarker with self-reported diet data) ${ }^{(10,11)}$, integration of validated biomarkers into epidemiological studies, and the development and validation of new biomarkers that characterize dietary components ${ }^{(12,13)}$. Nutrient biomarkers have been classified as recovery, concentration, predictive or replacement ${ }^{(14)}$, depending upon whether the biomarker reflects an absolute level of intake or is correlated with dietary intake (i.e. recovery $v$. concentration), the degree to which the biomarker is recovered and quantifiable (i.e. predictive) ${ }^{(15)}$ or is used as a surrogate measure of intake for nutrients difficult to assess or with no food composition data available (i.e. replacement).

Methodological approaches for incorporating biomarkers within epidemiological studies have been developed ${ }^{(10,11,16)}$ and applications of these approaches have strengthened associations in diet-disease analyse ${ }^{(17-20)}$. With combined biomarker and self-reported dietary data, the sample size requirement for estimating diet-disease associations may be reduced by $20-50 \%$ compared with self-reported intake alone $^{(11)}$. A predictive biomarker for total sugars intake (i.e. sum of fructose and sucrose in $24 \mathrm{~h}$ urine) developed in two controlled feeding studies in the UK showed that the sum of urinary sucrose and fructose in $24 \mathrm{~h}$ urine was significantly correlated with total sugars $(r=0.841, P<0 \cdot 001)$ and sucrose intake $(r=0.773, P=0.002)^{(15)}$. This biomarker has been recently integrated into two US-based biomarker studies with free-living individuals as a reference instrument against FFQ-, 24HR- and food record-based sugars intake ${ }^{(21,22)}$. The objective of the present study was to compare the consumption of sugars estimated from self-report with values derived from a biomarker of sugars intake nested within a large observational cohort study of Hispanic/Latino adults living in the USA.

\section{Methods}

\section{Study description}

The Study of Latinos: Nutrition \& Physical Activity Assessment Study (SOLNAS), an ancillary study of the Hispanic Community Health Study/Study of Latinos (HCHS/SOL), included Hispanic/Latino men and women aged 18 to 74 years at HCHS/SOL baseline who were recruited from four centres (Bronx, NY; Chicago, IL; Miami, FL; San Diego, CA) across the USA, as previously described ${ }^{(23-25)}$. After excluding SOLNAS participants having incomplete $(<500 \mathrm{ml} / \mathrm{d}$ ) or missing urine samples ( $n 26$ for the primary study and $n 6$ for the reliability study), the analytic sample was 450 for the primary sample and ninety for the reliability sample. Dietary recalls were excluded at each time point if reported daily energy intake was $<2510$ or $>12552 \mathrm{~kJ}$ ( $<600$ or $>3000 \mathrm{kcal}$ ) for women or $<3347$ or $>16736 \mathrm{~kJ}$ ( $<800$ or $>4000 \mathrm{kcal}$ ) for men (Fig. 1) ${ }^{(26)}$.

\section{Energy expenditure and self-reported physical activity assessment}

Energy expenditure was measured using a doubly labelled water (DLW) protocol ${ }^{(27)}$. Following the collection of a baseline urine sample, participants ingested a DLW mixture that provided $1.38 \mathrm{~g}$ of $10 \mathrm{at} \%{ }^{18} \mathrm{O}$-labelled water and $0.086 \mathrm{~g}$ of 99.9 at $\%{ }^{2} \mathrm{H}$-labelled water per kilogram body weight and provided in-clinic spot urines at 3 and $4 \mathrm{~h}^{(28)}$. Participants aged $\geq 60$ years provided a blood sample $3 \mathrm{~h}$ post-isotope to allow adjustment for age-related post-void urine retention. An additional post-dose sample was collected on day 12 of the DLW protocol. Self-reported

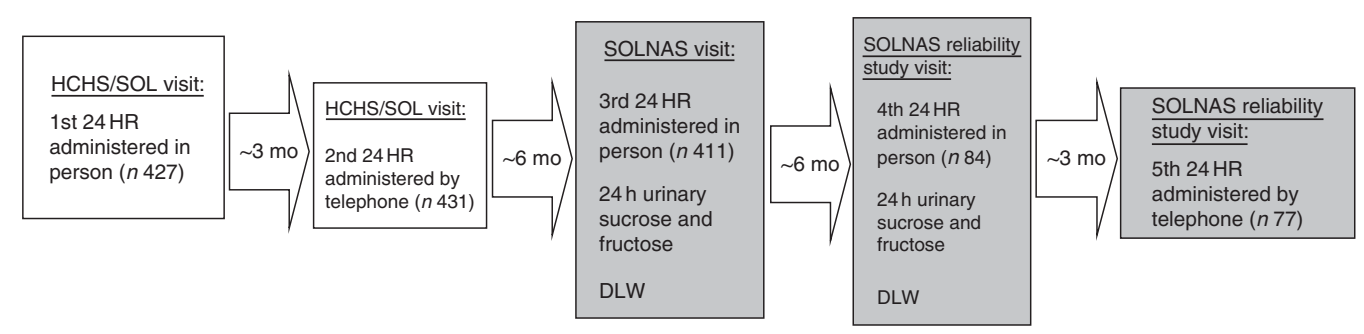

Fig. 1 Study flow diagram: estimating self-reported dietary intake using the National Cancer Institute (NCl) method and objective dietary intake using biomarkers of energy and sugars intake within the Study of Latinos: Nutrition \& Physical Activity Assessment Study (SOLNAS), an ancillary study of the Hispanic Community Health Study/Study of Latinos (HCHS/SOL) cohort. At each time point, $24 \mathrm{~h}$ recalls $(24 \mathrm{HR})$ were excluded if energy intake was $<2510$ or $>12552 \mathrm{~kJ}$ ( $<600$ or $>3000 \mathrm{kcal})$ for women or $<3347$ or $>16736 \mathrm{~kJ}$ ( $<800$ or $>4000 \mathrm{kcal})$ for men. Combining recalls using the $\mathrm{NCl}$ method, the analytic sample size was 450 for the primary study and ninety for the reliability study. For deriving usual intake, 24HR from the entire HCHS/SOL were used ( $n 15622$ for visit 1 and $n 14709$ for visit 2). $n$, sample size; mo, months; DLW, doubly labelled water 
physical activity was assessed by the Global Physical Activity Questionnaire that was developed by the WHO to quantify time spent in moderate and vigorous levels of physical activity at work, travel and leisure time ${ }^{(29,30)}$. Twenty per cent of the participants ( $n$ 96) repeated the protocol to obtain reliability measures.

\section{Self-reported sugars intake assessment}

Self-reported sugars intake was estimated using the National Cancer Institute (NCI) method ${ }^{(31)}$, using all available data to estimate usual dietary intake by combining up to five $24 \mathrm{HR}$ recalls (Fig. 1). In-person $24 \mathrm{HR}$ were conducted at the HCHS/SOL baseline, SOLNAS baseline and the SOLNAS reliability study visit (see online supplementary material, Supplemental Table 1). Interviews were conducted in Spanish or English depending on the participant's preference with the Nutrition Data System for Research (NDS-R) software (version 11) developed by the Nutrition Coordinating Center at the University of Minnesota, which uses the multiple-pass method and has a database with >18000 foods. As described by Tooze et al. ${ }^{(32)}$, the NCI method for estimating usual intake involved two steps. The first step (NCI MIXTRAN macro) specifies the consumption-day amount using linear regression on a transformed scale, with a person-specific effect adjusted for sex, age, Hispanic/ Latino background, field centre, weekend (including Friday), self-reported intake amount (more, same or less than usual amount) and sequence (i.e. Fig. 1, first through fifth recall). The second step (NCI INDIVINT macro) calculates the individual's predicted usual intake using parameter estimates from the first step.

\section{Biomarker-predicted energy assessment}

The urine and plasma samples collected within the DLW protocol from SOLNAS participants were analysed by gas-isotope-ratio MS to assess energy expenditure ${ }^{(33)}$. The isotopic data were converted to energy expenditure values based on an energy equivalent of 1 litre of $\mathrm{CO}_{2}$ to be 3.815/ $\mathrm{RQ}+1 \cdot 2321$, where RQ is the respiratory quotient equal to $0 \cdot 86$, a standard among populations consuming a Western diet which is based on a high-fat diet ${ }^{(25,34)}$.

\section{Biomarker-predicted sugars assessment}

At SOLNAS baseline, participants collected one $24 \mathrm{~h}$ urine sample that was analysed for sucrose and fructose. Urinary sucrose and fructose were measured by LC-MS at the University of Hawaii Cancer Center ${ }^{(35)}$. Urine samples $(20 \mu \mathrm{l})$ mixed with internal standards were dried using $\mathrm{N}_{2}$ and reconstituted in $100 \mu \mathrm{l} \mathrm{MeOH}$. The redissolved sample was centrifuged and the supernatant $(10 \mu \mathrm{l})$ was injected into the LC-MS system (model Accela ultra HPLC coupled to a TSQ Quantum Ultra tandem mass spectrometer with Xcalibur $^{\mathrm{TM}}$ software; ThermoFisher, San Jose, CA, USA). Chromatographic separations were performed on a
ZIC $^{\circledR}$-HILIC column $(100 \mathrm{~mm} \times 2 \cdot 1 \mathrm{~mm}, 3 \mu \mathrm{m}$; Merck KGaA, Darmstadt, Germany) by gradient elution using $0 \cdot 1 \%(\mathrm{v} / \mathrm{v})$ formic acid in MeCN and $0.1 \%(v / v)$ formic acid in $\mathrm{H}_{2} \mathrm{O}$ at a flow rate of $0.3 \mathrm{ml} / \mathrm{min}$. Masses were continuously monitored by atmospheric-pressure chemical ionization in negative mode and selected ion monitoring by extracting the respective accurate mass-to-charge $(\mathrm{m} / \mathrm{z})$ ratios.

Among a $10 \%$ blinded quality control sample (collected about once per month from among SOLNAS $24 \mathrm{~h}$ urine samples; $n$ 50), the CV were $11.7 \%$ for fructose and $8.0 \%$ for sucrose. Per an internal laboratory quality control ( $n$ 11), intra-day CV were $4.6 \%$ for fructose and $5.8 \%$ for sucrose, and inter-day CV were $10.5 \%$ for both fructose and sucrose.

We used the calibration equation (1) below for total sugars biomarker, previously developed based on data from a feeding study ${ }^{(15,21)}$, to calibrate the biomarker (i.e. sum of $24 \mathrm{~h}$ urine sucrose and fructose) and to derive biomarkerpredicted sugars (BPS) intake in SOLNAS participants:

$$
P M_{i j}=M_{i j}-1 \cdot 67-0 \cdot 02 \times S_{i}+0.71 \times A_{i},
$$

where:

$P M_{i j}=\log$-transformed calibrated biomarker, i.e. BPS intake, for individual $i$ on day $j$;

$M_{i j}=\log$-transformed (sum of $24 \mathrm{~h}$ urine fructose and sucrose) for individual $i$ on day $j$;

$S_{i}=$ sex of individual $i$ ( 0 for men, 1 for women); and

$A_{i}=\log$-transformed age of individual $i$.

\section{Statistical analysis}

Both self-reported sugars intake and BPS intake were logtransformed to improve normality. Geometric means and 95\% CI were computed for self-reported sugars and BPS intakes, overall and by selected participant characteristics. Participant characteristics (mean and SD for continuous variables; $n$ and \% for categorical variables) were summarized by quartile of BPS intake. We assessed the correlations of BPS intake $(\mathrm{g} / \mathrm{d})$ with self-reported sugars intake $(\mathrm{g} / \mathrm{d})$ using Spearman correlation coefficients. Among the reliability participants, Spearman correlations were calculated to assess the relationship between repeated measures of selfreported and BPS intake.

To examine the sensitivity of the results to the analytic approaches used, results were stratified by accuracy of reporting status, with 'concordance' defined as selfreported energy intake within $25 \%$ of energy expenditure estimated by DLW. Analyses were repeated using the 'raw' sum of $24 \mathrm{~h}$ urine fructose and sucrose (i.e. uncalibrated biomarker), rather than using BPS (i.e. calibrated biomarkers), as a measure of objective sugars intake. Furthermore, BPS was correlated to selfreported estimates of total sugars intake from a single $24 \mathrm{HR}$ recall which corresponded to the time point closest to the urine collection (e.g. $24 \mathrm{HR}$ administered within $7 \mathrm{~d}$ of $24 \mathrm{~h}$ urine collection), rather than using the NCI method 
to estimate usual intake, as the measure of sugars intake. Statistical analyses were conducted using the statistical software package SAS version 9.3.

\section{Results}

Overall, geometric mean self-reported total sugars intake was $90 \cdot 6$ (95\% CI 87.6, 93.6) g/d $v .167 \cdot 5$ (95\% CI 154.4, 181.7) g/d for biomarker-predicted total sugars intake (Table 1). Whereas self-reported total sugars intake was not associated with participant characteristics, BPS intake was significantly associated with age and ethnicity (Table 2). There was a nonsignificant trend for a higher proportion of obese individuals and those with lower education level to be in the highest BPS quartile. BPS intake was also higher among older participants and Puerto Ricans. Self-reported total sugars intake was not correlated with BPS $(r=-0 \cdot 06, P=0 \cdot 20)$.

The self-reported total sugars intake and BPS intake were not related, irrespective of whether energy expenditure estimated by DLW was within $25 \%$ of self-reported total energy intake ( $P>0.05$; Table 3$)$. Usual energy intake was correlated with energy expenditure measured with the DLW method, and it was more highly correlated among true reporters compared with participants who were not classified as concordant reporters $(r=0.79 v \cdot r=0.54, P<0.0001)$. Among the participants in the $20 \%$ reliability sub-samples who repeated the entire protocol about 6 months after the SOLNAS baseline visit, the repeated measures of BPS intake at baseline and 6 months were more highly correlated than repeated self-reported total sugars intake $(r=0.59 v, r=0.20$; for gender-specific reliability coefficients see Fig. 2).

Sensitivity analyses also demonstrated the lack of an association between urinary fructose and sucrose and self-reported total sugars intake. Among the primary study participants, the correlation between the 'raw'/ uncalibrated sum of $24 \mathrm{HR}$ urinary fructose and sucrose and NCI-based sugars intake was $r=0.03(P=0.58)$ and the agreement between quartiles of the raw sum and BPS was high $(\kappa=0.72, P<0.0001$; see online supplementary material, Supplemental Table 2). Similarly, the correlation between BPS and a single $24 \mathrm{HR}$ corresponding to the time point closest to the urine collection as the measure of self-reported total sugars intake, rather than using the NCI method to estimate usual intake, was $r=0.02(P=0 \cdot 70)$. Within the reliability study, the correlation between BPS and a single $24 \mathrm{HR}$ corresponding to the time point closest to the urine collection was $r=0.36 \quad(P<0.0001)$; the association was stronger in men $(r=0 \cdot 63, P<0 \cdot 001)$ than in women $(r=0 \cdot 27, P=0 \cdot 04$; Supplemental Table 3$)$.

Table 1 Geometric mean $(95 \% \mathrm{Cl})$ of self-reported total sugars and biomarker-predicted sugars intake ( $n$ 450); Study of Latinos: Nutrition \& Physical Activity Assessment Study (SOLNAS)

\begin{tabular}{|c|c|c|c|c|c|}
\hline & \multirow[b]{2}{*}{$n$} & \multicolumn{2}{|c|}{ Self-reported sugars intake $(\mathrm{g} / \mathrm{d})^{*}$} & \multicolumn{2}{|c|}{ Biomarker-predicted sugars intake $(\mathrm{g} / \mathrm{d})^{\star}$} \\
\hline & & Mean & $95 \% \mathrm{Cl}$ & Mean & $95 \% \mathrm{Cl}$ \\
\hline Overall & 450 & $90 \cdot 6$ & $87 \cdot 6,93 \cdot 6$ & $167 \cdot 5$ & $154 \cdot 4,181 \cdot 7$ \\
\hline \multicolumn{6}{|l|}{ Age (years) } \\
\hline $18-44$ & 172 & 95.2 & $90 \cdot 4,100 \cdot 3$ & $116 \cdot 2$ & $101 \cdot 5,133 \cdot 0$ \\
\hline $45-64$ & 252 & 88.4 & $84 \cdot 5,92 \cdot 5$ & $205 \cdot 6$ & $186 \cdot 6,226 \cdot 6$ \\
\hline $65+$ & 26 & $82 \cdot 0$ & $72 \cdot 0,93 \cdot 4$ & $257 \cdot 1$ & $183 \cdot 9,359 \cdot 3$ \\
\hline \multicolumn{6}{|l|}{ Sex } \\
\hline Women & 276 & 83.5 & $80 \cdot 5,86 \cdot 6$ & $157 \cdot 6$ & $142 \cdot 8,174 \cdot 0$ \\
\hline Men & 174 & $103 \cdot 0$ & $97 \cdot 0,109 \cdot 2$ & 184.3 & $160 \cdot 0,212 \cdot 3$ \\
\hline \multicolumn{6}{|l|}{ Language of interview } \\
\hline English & 105 & $99 \cdot 1$ & $92 \cdot 4,106 \cdot 3$ & $155 \cdot 3$ & $129 \cdot 4,186 \cdot 3$ \\
\hline Spanish & 345 & $88 \cdot 1$ & $84.9,91.4$ & 171.3 & $156 \cdot 4,187 \cdot 7$ \\
\hline \multicolumn{6}{|l|}{ Weight status } \\
\hline Underweight $\left(<18.5 \mathrm{~kg} / \mathrm{m}^{2}\right)$ & 4 & $80 \cdot 8$ & $44 \cdot 7,146 \cdot 1$ & $203 \cdot 0$ & $83 \cdot 8,492 \cdot 0$ \\
\hline Normal $\left(18.5-24.9 \mathrm{~kg} / \mathrm{m}^{2}\right)$ & 85 & 101.9 & $95 \cdot 2,109.1$ & 135.4 & $112 \cdot 4,163 \cdot 1$ \\
\hline Overweight $\left(25.0-29.9 \mathrm{~kg} / \mathrm{m}^{2}\right)$ & 180 & 87.8 & $83 \cdot 6,92 \cdot 3$ & $171 \cdot 2$ & $151.9,193.0$ \\
\hline Obese $\left(\geq 30.0 \mathrm{~kg} / \mathrm{m}^{2}\right)$ & 181 & 88.5 & $83 \cdot 7,93 \cdot 7$ & $180 \cdot 2$ & $157 \cdot 0,207 \cdot 0$ \\
\hline \multicolumn{6}{|l|}{ Hispanic/Latino background } \\
\hline Central American & 50 & $87 \cdot 6$ & $79 \cdot 0,97 \cdot 2$ & $170 \cdot 6$ & $129 \cdot 9,224 \cdot 1$ \\
\hline Cuban & 65 & $82 \cdot 4$ & $75 \cdot 6,89 \cdot 8$ & $186 \cdot 1$ & $152 \cdot 7,226 \cdot 8$ \\
\hline Dominican & 47 & $70 \cdot 9$ & $64.5,78.0$ & $138 \cdot 2$ & $97 \cdot 6,195 \cdot 8$ \\
\hline Mexican & 135 & 98.8 & $93 \cdot 8,104 \cdot 1$ & $153 \cdot 3$ & $133 \cdot 1,176 \cdot 7$ \\
\hline Puerto Rican & 115 & 94.0 & $87 \cdot 6,100 \cdot 9$ & 198.9 & $170 \cdot 1,232 \cdot 6$ \\
\hline South American & 38 & $98 \cdot 3$ & $88.3,109.5$ & $140 \cdot 3$ & $114.5,171.9$ \\
\hline \multicolumn{6}{|l|}{ Income } \\
\hline Low income (<\$US 30 000/year) & 299 & 91.3 & $87 \cdot 7,95 \cdot 1$ & $172 \cdot 8$ & $156 \cdot 5,190 \cdot 7$ \\
\hline High income ( $\geq \$ \cup S 30000 /$ year) & 119 & $90 \cdot 7$ & $85 \cdot 0,96 \cdot 8$ & $157 \cdot 7$ & $133 \cdot 9,185 \cdot 6$ \\
\hline Missing & 32 & 83.0 & $73 \cdot 3,94 \cdot 1$ & $156 \cdot 4$ & $112 \cdot 2,218 \cdot 0$ \\
\hline \multicolumn{6}{|l|}{ Education } \\
\hline Less than high school & 143 & 89.8 & $84 \cdot 7,95 \cdot 2$ & $199 \cdot 1$ & $170 \cdot 8,232 \cdot 1$ \\
\hline High school equivalent & 112 & 93.5 & $87 \cdot 1,100 \cdot 5$ & 163.6 & $139.5,191.9$ \\
\hline Greater than high school & 195 & 89.4 & $85 \cdot 2,93 \cdot 9$ & $149 \cdot 4$ & $132 \cdot 7,168 \cdot 3$ \\
\hline
\end{tabular}


Table 2 Characteristics of participants by quartile of biomarker-predicted total sugars intake ( $n$ 450); Study of Latinos: Nutrition \& Physical Activity Assessment Study (SOLNAS)

\begin{tabular}{|c|c|c|c|c|c|c|c|c|c|}
\hline \multirow[b]{2}{*}{ Characteristic } & \multicolumn{2}{|c|}{ Q1 ( $n$ 113) } & \multicolumn{2}{|c|}{ Q2 ( $n$ 112) } & \multicolumn{2}{|c|}{ Q3 (n 112) } & \multicolumn{2}{|c|}{ Q4 ( $n$ 112) } & \multirow[b]{2}{*}{$P$ value } \\
\hline & Mean & SD & Mean & SD & Mean & SD & Mean & SD & \\
\hline $\begin{array}{l}\text { Biomarker-predicted total sugars intake }(\mathrm{g} / \mathrm{d}) \text {, range } \\
\text { Self-reported intake, } \mathrm{NCl} \text { method }\end{array}$ & \multicolumn{2}{|c|}{$6 \cdot 5-104 \cdot 5$} & \multicolumn{2}{|c|}{$104 \cdot 7-168 \cdot 3$} & \multicolumn{2}{|c|}{$169 \cdot 4-276 \cdot 0$} & \multicolumn{2}{|c|}{$277 \cdot 8-2921 \cdot 2$} & \\
\hline Total sugars intake $(\mathrm{g} / \mathrm{d})$ & 98 & 32 & 96 & 34 & 97 & 39 & 95 & 35 & 0.9 \\
\hline Energy intake $(\mathrm{kJ} / \mathrm{d})$ & 8163 & 1992 & 7971 & 2050 & 7883 & 2138 & 7816 & 2059 & 0.41 \\
\hline Energy intake $(\mathrm{kcal} / \mathrm{d})$ & 1951 & 476 & 1905 & 490 & 1884 & 511 & 1868 & 492 & 0.41 \\
\hline Energy expenditure by DLW (kJ/d) & 9908 & 2013 & 9908 & 2192 & 10205 & 2109 & 10535 & 2276 & $0 \cdot 10$ \\
\hline Energy expenditure by DLW (kcal/d) & 2368 & 481 & 2368 & 524 & 2439 & 504 & 2518 & 544 & $0 \cdot 10$ \\
\hline \multirow[t]{2}{*}{ Age (years) } & 39 & 13 & 46 & 12 & 48 & 12 & 52 & 11 & $<0.0001$ \\
\hline & $n$ & $\%$ & $n$ & $\%$ & $n$ & $\%$ & $n$ & $\%$ & \\
\hline \multicolumn{9}{|l|}{ Gender } & 0.54 \\
\hline Female & 74 & 65.5 & 71 & 63.4 & 67 & $59 \cdot 8$ & 64 & $56 \cdot 6$ & \\
\hline Male & 39 & 34.5 & 41 & $36 \cdot 6$ & 45 & $40 \cdot 2$ & 49 & 43.4 & \\
\hline \multicolumn{9}{|l|}{ Weight status } & 0.41 \\
\hline Underweight $\left(<18.5 \mathrm{~kg} / \mathrm{m}^{2}\right)$ & 0 & 0.0 & 1 & 0.9 & 2 & 1.8 & 1 & 0.9 & \\
\hline Normal $\left(18.5-24.9 \mathrm{~kg} / \mathrm{m}^{2}\right)$ & 28 & $24 \cdot 8$ & 25 & $22 \cdot 3$ & 16 & $14 \cdot 3$ & 16 & $14 \cdot 2$ & \\
\hline Overweight $\left(25.0-29.9 \mathrm{~kg} / \mathrm{m}^{2}\right)$ & 43 & 38.1 & 45 & $40 \cdot 2$ & 48 & $42 \cdot 9$ & 44 & 38.9 & \\
\hline Obese $\left(\geq 30.0 \mathrm{~kg} / \mathrm{m}^{2}\right)$ & 42 & $37 \cdot 2$ & 41 & $36 \cdot 6$ & 46 & $41 \cdot 1$ & 52 & $46 \cdot 0$ & \\
\hline \multicolumn{9}{|l|}{ Hispanic/Latino background } & 0.04 \\
\hline Central American & 16 & $14 \cdot 2$ & 10 & 8.9 & 10 & 8.9 & 11 & 9.7 & \\
\hline Cuban & 15 & $13 \cdot 3$ & 11 & 9.8 & 11 & 9.8 & 13 & 11.5 & \\
\hline Dominican & 15 & $13 \cdot 3$ & 10 & 8.9 & 18 & $16 \cdot 1$ & 22 & 19.5 & \\
\hline Mexican & 37 & $32 \cdot 7$ & 39 & $34 \cdot 8$ & 34 & $30 \cdot 4$ & 25 & $22 \cdot 1$ & \\
\hline Puerto Rican & 21 & $18 \cdot 6$ & 26 & $23 \cdot 2$ & 29 & $25 \cdot 9$ & 39 & 34.5 & \\
\hline South American & 9 & 8.0 & 16 & $14 \cdot 3$ & 10 & 8.9 & 3 & $2 \cdot 7$ & \\
\hline \multicolumn{9}{|l|}{ Income } & 0.36 \\
\hline Low income (<\$US 30 000/year) & 74 & 65.5 & 65 & $58 \cdot 0$ & 81 & $72 \cdot 3$ & 79 & $69 \cdot 9$ & \\
\hline High income ( $\geq \$$ US 30 000/year) & 29 & $25 \cdot 7$ & 38 & 33.9 & 25 & $22 \cdot 3$ & 27 & 23.9 & \\
\hline Missing & 10 & 8.9 & 9 & 8.0 & 6 & 5.4 & 7 & $6 \cdot 2$ & \\
\hline \multicolumn{9}{|l|}{ Educational level } & 0.56 \\
\hline Less than high school & 32 & $28 \cdot 3$ & 31 & $27 \cdot 7$ & 37 & 33.0 & 43 & $38 \cdot 1$ & \\
\hline High school equivalent & 26 & 23.0 & 31 & $27 \cdot 7$ & 30 & $26 \cdot 8$ & 25 & $22 \cdot 1$ & \\
\hline Greater than high school & 55 & 48.7 & 50 & 44.6 & 45 & $40 \cdot 2$ & 45 & $39 \cdot 8$ & \\
\hline \multicolumn{9}{|l|}{ Smoking } & 0.22 \\
\hline Never & 79 & 69.9 & 66 & 58.9 & 65 & $58 \cdot 0$ & 58 & $51 \cdot 8$ & \\
\hline Past & 17 & $15 \cdot 0$ & 22 & $19 \cdot 6$ & 22 & $19 \cdot 6$ & 28 & $25 \cdot 0$ & \\
\hline Current & 17 & $15 \cdot 0$ & 24 & $21 \cdot 4$ & 25 & $22 \cdot 3$ & 26 & $23 \cdot 2$ & \\
\hline Self-reported physical activity ${ }^{\star}$ (min/week) & 439 & 684 & 580 & 1019 & 675 & 993 & 461 & 735 & 0.15 \\
\hline
\end{tabular}

DLW, doubly labelled water.

*Self-reported physical activity is the total amount of time spent doing some form of physical activity for work, transportation, recreation and sedentary behaviour in a week from the modified Global Physical Activity Questionnaire (GPAQ; available at https://www2.cscc.unc.edu/hchs/system/files/forms/UNLICOMM PhysicalPAE02182008.pdf).

Table 3 Spearman correlations between self-reported and biomarker-based intakes of energy and sugars by concordance with doubly labelled water*

\begin{tabular}{lccccr}
\hline Concordance & $n$ & $\begin{array}{c}\text { Energy intake } \\
(\mathrm{kcal} / \mathrm{d})\end{array}$ & $P$ value & $\begin{array}{c}\text { Total sugars } \\
(\mathrm{g} / \mathrm{d})\end{array}$ & $P$ value \\
\hline Concordant & 234 & 0.79 & $<0.0001$ & 0.04 & 0.59 \\
Discordant & 210 & 0.54 & $<0.0001$ & -0.06 & 0.37 \\
\hline
\end{tabular}

${ }^{*}$ Concordance $=$ reported energy intake within $25 \%$ of energy intake measured with the doubly labelled water method.

\section{Discussion}

Among a sizeable, diverse sample of Hispanics/Latinos, BPS intake was not correlated with self-reported total sugars intake. Whereas BPS was correlated with age and ethnicity, self-reported total sugars intake was unrelated to participant characteristics. Contrary to expectation, there was no significant association between BMI and BPS ${ }^{(36)}$. Using the sugars biomarker measured in spot urines, the European Prospective Investigation into Cancer and Nutrition-Norfolk reported positive associations between sucrose intake and obesity ${ }^{(37,38)}$ and a randomized, crossover trial in ten normal-weight and nine overweight/ obese participants suggested BMI does not affect the 

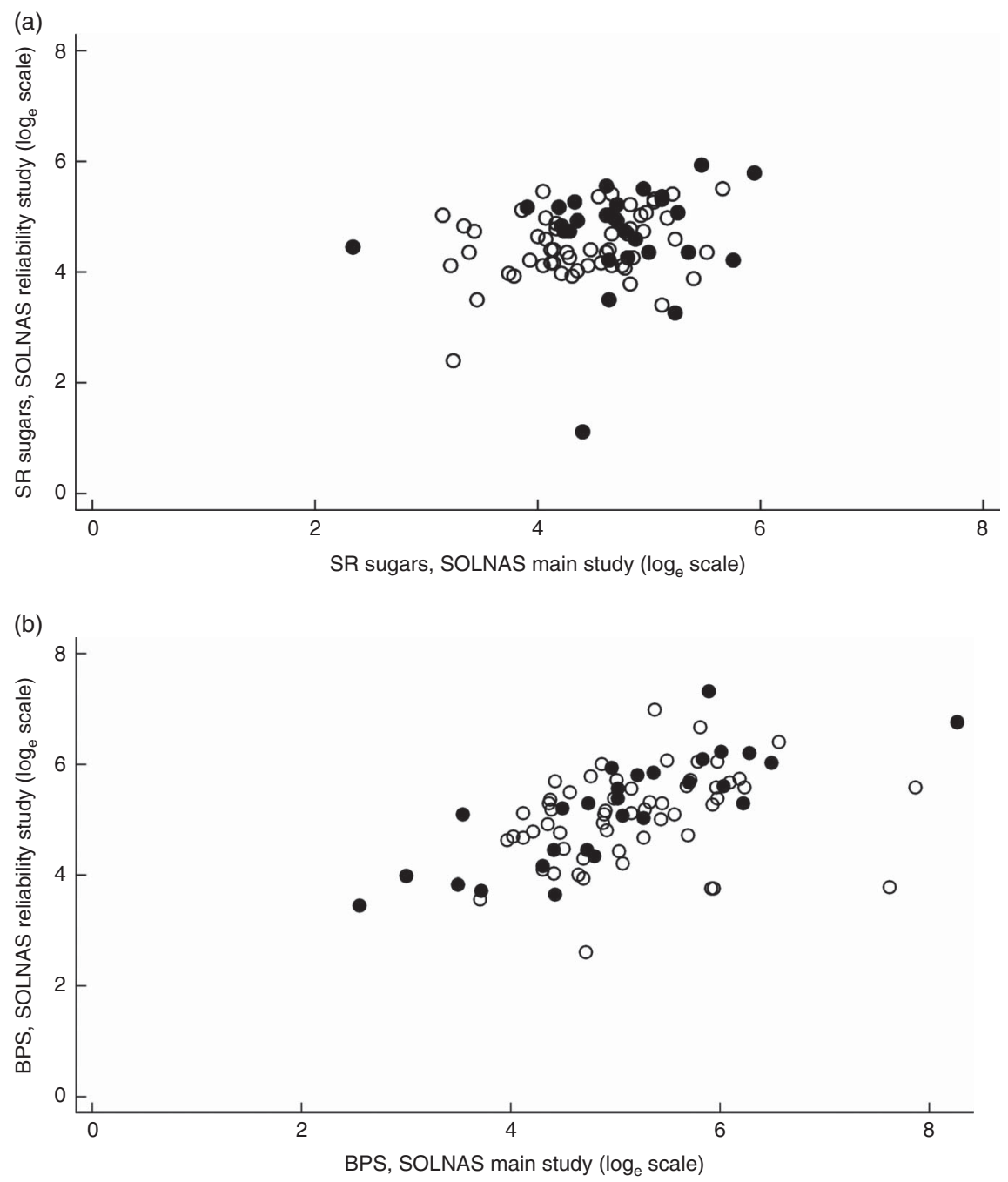

Fig. 2 Correlation of (a) self-reported (SR) total sugars intake and (b) biomarker-predicted sugars (BPS) intake, by sex (O, women; , men), between participants in the reliability sub-sample and participants in the main study; Study of Latinos: Nutrition \& Physical Activity Assessment Study (SOLNAS). (a) Women ( $n$ 58): Spearman's $\rho=0.21, P=0.11$; men ( $n$ 32): Spearman's $\rho=0.12$, $P=0.50$. (b) Women ( $n$ 57): Spearman's $\rho=0.42, P=0.001$; men $(n$ 32), Spearman's $\rho=0.84, P<0.0001$

validity of the biomarker ${ }^{(39)}$. As this biomarker, so far, has not been validated for use among Hispanics/Latinos, we cannot definitively quantify the measurement error in the self-reported total sugars intake $v$. BPS intake. However, we conducted further analyses to better understand the reasons for the observed low correlations between self-reported and BPS intake. Using the recovery biomarker for energy intake based on DLW data, just over half of the sample (53\%) was categorized as concordant (i.e. self-reported energy intake values were within $25 \%$ of biomarker values), but there was no association between self-reported total sugars and BPS intakes when results were restricted to this subset.

Possible explanations for the lack of any association between BPS and self-reported sugars intake include measurement error in self-reported sugars intake, variability in sugars intake necessitating multiple $24 \mathrm{HR}$ recalls and measures of urinary sucrose and fructose to accurately estimate usual intake, and/or the lack of evidence to support the role of urinary sucrose and fructose as a valid proxy for total sugars intake in this study population. Measurement error in the reporting of energy and protein has been well established in several studies comparing self-reported intake with recovery biomarkers ${ }^{(5,10,16)}$. Other studies have nested the predictive urinary sucrose and fructose biomarker into validation studies to compare self-reported intake and biomarker-predicted intake of total sugars ${ }^{(21)}$. In the Women's Health Initiative Nutrition and Physical Activity Assessment Study (NPAAS; $n$ 450), self-reported sugars intake was substantially, and roughly equally, misreported whether measured by FFQ, $4 \mathrm{~d}$ food record or $24 \mathrm{HR}$ recall ${ }^{(21)}$. Geometric means of BPS in NPAAS and SOLNAS were similar: $173.9(95 \% \mathrm{CI}$ 142.9, 211.6) g/d $v .167 .5$ (95\% CI 154.4, 181.7) g/d, respectively ${ }^{(21)}$. The biomarker-prediction equation used in ours and each of these validation studies was derived 
from a highly controlled feeding study among seven men and six women in the UK aged $23-66$ years ${ }^{(15,21,40)}$. We noted a strong positive association between the BPS and age (Table 1), which may be due to an overcorrection for age at the higher age ranges. In analyses where we did not apply the biomarker-prediction equation and relied on the sum of urinary sucrose and fructose, associations between age and self-reported intake were null. Data from a controlled feeding study including participants representative of the age, race/ethnicity and BMI of this cohort would inform whether the biomarker-prediction equation is generalizable or needs modification based on these or other participant characteristics.

Whereas recovery biomarkers (i.e. DLW and $24 \mathrm{~h}$ urinary-N) are unbiased reference instruments that reflect an absolute level of intake, predictive biomarkers, such as $24 \mathrm{~h}$ urinary sucrose and fructose, can also be used as reference validation instruments after being calibrated to account for bias in the biomarker, estimable from a feeding study against known intake ${ }^{(15)}$. We did not observe significant correlations between self-reported sugars intake and sucrose and fructose based on urinary measurements, even when restricting the analysis to individuals who reported energy intake within $25 \%$ of the DLW value. Another possibility for the lack of correlation between self-reported and BPS intake is that high intraindividual variation in sugars intake would necessitate multiple days of measurement in order to estimate usual intake of total sugars. Among the sub-sample of reliability study participants, the reproducibility of self-reported sugars intake was much lower than the reproducibility of the urinary sucrose and fructose biomarkers. Furthermore, the self-reported intake from the $24 \mathrm{HR}$ closest to the urine collection and BPS was significant among reliability participants ( $r=0.36, P<0 \cdot 001, n 84)$. However, restricting the analysis to individuals having $24 \mathrm{HR}$ within one week of the urinary sucrose and fructose biomarker measurement did not result in significant correlations with self-reported sugars intake. Possible explanations include that the reliability participants were more accurate reporters of dietary intake and more compliant with the urine collection protocols compared with the rest of the SOLNAS participants. Since the equation for predicting biomarker-based sugars intake was developed based on a small sample of individuals in the UK, we examined correlations between both the 'raw' sum of urinary sucrose and fructose in addition to applying the biomarkerprediction equation, but this did not substantively alter our results.

Strengths of the current study include applying a predictive biomarker that has been validated in controlled feeding studies to an ethnically diverse cohort, representing both genders, of Hispanics/Latinos in the USA, as well as accounting for a wide range of other factors previously demonstrated to be associated with measurement error in self-reported diet intake, such as age and BMI.
The substantial sample size allowed conduct of several sensitivity analyses to ascertain whether our findings were influenced by the characterization of self-reported intake (i.e. usual intake per NCI method or restricting to $24 \mathrm{HR}$ within one week of the $24 \mathrm{~h}$ urine collection time point) and the level of concordance between DLW and selfreported energy intake. Our ability to make inferences about the magnitude of measurement error in selfreported sugars intake using the biomarker in this study population is limited. Highly controlled feeding studies with participants representative of the HCHS/SOL population would better characterize the application of this biomarker among Hispanics/Latinos.

\section{Conclusion}

In conclusion, in comparing a predictive biomarker of sugars intake among a diverse sample of Hispanics/ Latinos, no significant associations were detected between the self-reported and biomarker-predicted sugars intakes. Clinical studies that allow for the control of factors such as the amount of total sugars intake, the optimal time frame between sugars intake and biomarker measurement, and health are needed to better determine the potential use of urinary sucrose and fructose as a biomarker of total sugars intake.

\section{Acknowledgements}

Acknowledgements: The authors would like to acknowledge Laurie J. Custer at the University of Hawaii for her LCMS work. The authors also thank the staff and participants of the Hispanic Community Health Study/Study of Latinos (HCHS/SOL) for their important contributions (investigators' website: http://www.cscc.unc.edu/hchs/). Financial support: The Sackler Institute for Nutrition Sciences funded this study. The Study of Latinos Nutrition \& Physical Activity Assessment Study (SOLNAS) was supported by the National Heart, Lung, and Blood Institute (NHLBI; grant \#11414319-R01HL095856). The HCHS/SOL was carried out as a collaborative study supported by contracts from the NHLBI to the University of North Carolina (N01-HC65233), the University of Miami (N01-HC65234), Albert Einstein College of Medicine (N01-HC65235), Northwestern University (N01-HC65236) and San Diego State University (N01-HC65237). The following institutes/ centres/offices contribute to the HCHS/SOL through a transfer of funds to the NHLBI: the National Center on Minority Health and Health Disparities; the National Institute of Deafness and Other Communications Disorders; the National Institute of Dental and Craniofacial Research; the National Institute of Diabetes and Digestive and Kidney Diseases; the National Institute of Neurological Disorders and Stroke; and the Office of 
Dietary Supplements. Additional support at the Albert Einstein College of Medicine was provided from the Clinical \& Translational Science Award (grant number UL1 TR001073) from the National Center for Advancing Translational Sciences at the National Institutes of Health. The funders had no role in the design, analysis or writing of this article. Conflict of interest: The authors have no conflict of interest to disclose. Authorship: Y.M.-R. and J.M.B. designed the research; W.W.W., P.A.S., A.M.S.-R., D.S.-A., M.D.G., M.S., L.V.H., A.A.F. and Y.M.-R. conducted the research and provided essential materials; M.J., P.A.S., N.T., D.S.-A. and J.M.B. developed the analytic approach and analysed the data; and J.M.B., J.R.K., J.S., J.W.-R. and Y.M.-R. wrote the paper. All authors read and approved the final manuscript. Ethics of buman subject participation: This study was conducted according to the guidelines laid down in the Declaration of Helsinki and all procedures involving human subjects/patients were approved by the Albert Einstein College of Medicine Institutional Review Board. Written informed consent was obtained from all subjects.

\section{Supplementary material}

To view supplementary material for this article, please visit http://dx.doi.org/10.1017/S1368980016001580

\section{References}

1. Johnson RK, Appel LJ, Brands M et al. (2009) Dietary sugars intake and cardiovascular health: a scientific statement from the American Heart Association. Circulation 120 1011-1020.

2. Hu FB \& Malik VS (2010) Sugar-sweetened beverages and risk of obesity and type 2 diabetes: epidemiologic evidence. Physiol Behav 100, 47-54.

3. Han E \& Powell LM (2013) Consumption patterns of sugar-sweetened beverages in the United States. I Acad Nutr Diet 113, 43-53.

4. Schneiderman N, Llabre M, Cowie CC et al. (2014) Prevalence of diabetes among Hispanics/Latinos from diverse backgrounds: the Hispanic Community Health Study/Study of Latinos (HCHS/SOL). Diabetes Care 37. 2233-2239.

5. Subar AF, Kipnis V, Troiano RP et al. (2003) Using intake biomarkers to evaluate the extent of dietary misreporting in a large sample of adults: the OPEN Study. Am J Epidemiol 158, $1-13$.

6. Kipnis V, Subar AF, Midthune D et al. (2003) Structure of dietary measurement error: results of the OPEN biomarker study. Am J Epidemiol 158, 14-21.

7. Prentice RL, Sugar E, Wang CY et al. (2002) Research strategies and the use of nutrient biomarkers in studies of diet and chronic disease. Public Health Nutr 5, 977-984.

8. Lissner L, Troiano RP, Midthune D et al. (2007) OPEN about obesity: recovery biomarkers, dietary reporting errors and BMI. Int J Obes (Lond) 31, 956-961.

9. Freedman LS, Commins JM, Moler JE et al. (2014) Pooled results from 5 validation studies of dietary self-report instruments using recovery biomarkers for energy and protein intake. Am J Epidemiol 180, 172-188.
10. Neuhouser ML, Tinker L, Shaw PA et al. (2008) Use of recovery biomarkers to calibrate nutrient consumption selfreports in the Women's Health Initiative. Am J Epidemiol 167, 1247-1259.

11. Freedman LS, Kipnis V, Schatzkin A et al. (2010) Can we use biomarkers in combination with self-reports to strengthen the analysis of nutritional epidemiologic studies? Epidemiol Perspect Innov 7, 2.

12. Prentice RL, Huang Y, Tinker LF et al. (2009) Statistical aspects of the use of biomarkers in nutritional epidemiology research. Statist Biosci 1, 112-123.

13. Bingham SA (2006) Biomarkers in nutritional epidemiology. Public Health Nutr 5, 821-827.

14. Jenab M, Slimani N, Bictash M et al. (2009) Biomarkers in nutritional epidemiology: applications, needs and new horizons. Hum Genet 125, 507-525.

15. Tasevska N, Runswick SA, McTaggart A et al. (2005) Urinary sucrose and fructose as biomarkers for sugar consumption. Cancer Epidemiol Biomarkers Prev 14, 1287-1294.

16. Prentice RL, Mossavar-Rahmani Y, Huang Y et al. (2011) Evaluation and comparison of food records, recalls, and frequencies for energy and protein assessment by using recovery biomarkers. Am J Epidemiol 174, 591-603.

17. Prentice RL, Huang Y, Kuller LH et al. (2011) Biomarkercalibrated energy and protein consumption and cardiovascular disease risk among postmenopausal women. Epidemiology 22, 170-179.

18. Beasley JM, LaCroix AZ, Neuhouser ML et al. (2010) Protein intake and incident frailty in the Women's Health Initiative observational study. J Am Geriatr Soc 58, 1063-1071.

19. Beasley JM, Aragaki AK, LaCroix AZ et al. (2011) Higher biomarker-calibrated protein intake is not associated with impaired renal function in postmenopausal women. $J$ Nutr 141, 1502-1507.

20. Prentice RL, Shaw PA, Bingham SA et al. (2009) Biomarkercalibrated energy and protein consumption and increased cancer risk among postmenopausal women. Am J Epidemiol 169, 977-989.

21. Tasevska N, Midthune D, Tinker LF et al. (2014) Use of a urinary sugars biomarker to assess measurement error in self-reported sugars intake in the nutrition and physical activity assessment study (NPAAS). Cancer Epidemiol Biomarkers Prev 23, 2874-2883.

22. Tasevska N, Park Y, Jiao L et al. (2014) Sugars and risk of mortality in the NIH-AARP Diet and Health Study. Am J Clin Nutr 99, 1077-1088.

23. Sorlie PD, Aviles-Santa LM, Wassertheil-Smoller S et al. (2010) Design and implementation of the Hispanic Community Health Study/Study of Latinos. Ann Epidemiol 20, 629-641.

24. Siega-Riz AM, Sotres-Alvarez D, Ayala GX et al. (2014) Food-group and nutrient-density intakes by Hispanic and Latino backgrounds in the Hispanic Community Health Study/Study of Latinos. Am J Clin Nutr 99, $1487-1498$.

25. Mossavar-Rahmani Y, Shaw PA, Wong WW et al. (2015) Applying recovery biomarkers to calibrate self-report measures of energy and protein in the Hispanic Community Health Study/Study of Latinos. Am J Epidemiol 181, 996-1007.

26. Nielsen SJ \& Adair L (2007) An alternative to dietary data exclusions. J Am Diet Assoc 107, 792-799.

27. Klein PD, James WP, Wong WW et al. (1984) Calorimetric validation of the doubly-labelled water method for determination of energy expenditure in man. Hum Nutr Clin Nutr 38, 95-106.

28. Blanc S, Colligan AS, Trabulsi J et al. (2002) Influence of delayed isotopic equilibration in urine on the accuracy of the ${ }^{2} \mathrm{H}_{2}{ }^{18} \mathrm{O}$ method in the elderly. J Appl Physiol (1985) 92, 1036-1044. 
29. Bull FC, Maslin TS \& Armstrong T (2009) Global physical activity questionnaire (GPAQ): nine country reliability and validity study. I Phys Act Health 6, 790-804.

30. World Health Organization, Department of Chronic Diseases and Health Promotion (2013) Global Physical Activity Questionnaire (GPAQ) Analysis Guide. http://www.who.int/ chp/steps/resources/GPAQ_Analysis_Guide.pdf

31. Dodd KW, Guenther PM, Freedman LS et al. (2006) Statistical methods for estimating usual intake of nutrients and foods: a review of the theory. J Am Diet Assoc 106, $1640-1650$

32. Tooze JA, Kipnis V, Buckman DW et al. (2010) A mixedeffects model approach for estimating the distribution of usual intake of nutrients: the NCI method. Stat Med 29, 2857-2868.

33. Wong WW, Lee LS \& Klein PD (1987) Deuterium and oxygen18 measurements on microliter samples of urine, plasma, saliva, and human milk. Am J Clin Nutr 45, 905-913.

34. Ravussin E, Harper IT, Rising R et al. (1991) Energy expenditure by doubly labeled water: validation in lean and obese subjects. Am J Physiol 261, E402-E409.

35. Kuhnle GG, Joosen AM, Wood TR et al. (2008) Detection and quantification of sucrose as dietary biomarker using gas chromatography and liquid chromatography with mass spectrometry. Rapid Commun Mass Spectrom 22, 279-282.

36. Tasevska N (2015) Urinary sugars - a biomarker of total sugars intake. Nutrients 7, 5816-5833.

37. Kuhnle GG, Tasevska N, Lentjes MA et al. (2015) Association between sucrose intake and risk of overweight and obesity in a prospective sub-cohort of the European Prospective Investigation into Cancer in Norfolk (EPICNorfolk). Public Health Nutr 18, 2815-2824.

38. Bingham S, Luben R, Welch A et al. (2007) Epidemiologic assessment of sugars consumption using biomarkers: comparisons of obese and nonobese individuals in the European prospective investigation of cancer Norfolk. Cancer Epidemiol Biomarkers Prev 16, 1651-1654.

39. Joosen AM, Kuhnle GG, Runswick SA et al. (2008) Urinary sucrose and fructose as biomarkers of sugar consumption: comparison of normal weight and obese volunteers. Int J Obes (Lond) 32, 1736-1740.

40. Tasevska N, Midthune D, Potischman N et al. (2011) Use of the predictive sugars biomarker to evaluate self-reported total sugars intake in the Observing Protein and Energy Nutrition (OPEN) study. Cancer Epidemiol Biomarkers Prev 20, 490-500. 